\title{
Ostrowski Inequalities for Functions Whose First Derivatives Are Logarithmically Preinvex
}

\author{
Badreddine Meftah \\ University of 8 May 1945 Guelma, P.O. Box 401, 24000 Guelma, Algeria \\ Correspondence should be addressed to Badreddine Meftah; badrimeftah@yahoo.fr
}

Received 28 April 2016; Revised 20 July 2016; Accepted 25 July 2016

Academic Editor: Annamaria Barbagallo

Copyright ( 2016 Badreddine Meftah. This is an open access article distributed under the Creative Commons Attribution License, which permits unrestricted use, distribution, and reproduction in any medium, provided the original work is properly cited.

Some Ostrowski type inequalities for functions whose first derivatives are logarithmically preinvex are established.

\section{Introduction}

In 1938, A. M. Ostrowski proved the following important inequality.

Theorem 1 (see [1]). Let $f: I \subseteq \mathbb{R} \rightarrow \mathbb{R}$ be a differentiable mapping on $I^{\circ}$ (interior of $I$ ), and let $a, b \in I^{\circ}$ with $a<b$. If $\left|f^{\prime}\right| \leq M$ for all $x \in(a, b)$, then

$$
\begin{aligned}
& \left|f(x)-\frac{1}{b-a} \int_{a}^{b} f(t) d t\right| \\
& \quad \leq M(b-a)\left[\frac{1}{4}+\frac{(x-(a+b) / 2)^{2}}{(b-a)^{2}}\right],
\end{aligned}
$$

$$
\forall x \in[a, b] .
$$

This is well known in the literature as Ostrowski's inequality. Due to its wide range of applications in numerical analysis and in probability, many researchers have established generalizations, extensions, and variants of inequality (1); we refer readers to [2-10] and the references cited therein.

In recent years, a lot of efforts have been made by many mathematicians to generalize classical convexity. Hanson [11] introduced a new class of generalized convexity, called invexity. In [12], the authors gave the concept of preinvex function which is a special case of invexity. Pini [13], Noor [14, 15], Yang and Li [16], and Weir and Mond [17] have studied the basic properties of the preinvex functions and their role in optimization, variational inequalities, and equilibrium problems.

In [5], Işcan established some Ostrowski type inequalities for functions whose derivatives in absolute value are preinvex, by using the following identity.

Lemma 2 (see [5]). Let $A \subset \mathbb{R}$ be an open invex subset with respect to $\eta: A \times A \rightarrow \mathbb{R}$ and $a, b \in A$ with $a<a+\eta(b, a)$. Suppose that $f: A \rightarrow \mathbb{R}$ is a differentiable function. If $f^{\prime}$ is integrable on $[a, a+\eta(b, a)]$, then the following equality holds:

$$
\begin{gathered}
f(x)-\frac{1}{\eta(b, a)} \int_{a}^{a+\eta(b, a)} f(u) d u=\eta(b, a) \\
\cdot\left(\int_{0}^{(x-a) / \eta(b, a)} t f^{\prime}(a+t \eta(b, a)) d t\right. \\
\left.+\int_{(x-a) / \eta(b, a)}^{1}(t-1) f^{\prime}(a+t \eta(b, a)) d t\right),
\end{gathered}
$$

for all $x \in[a, a+\eta(b, a)]$

Motivated by the results given in [5], in the present paper, we establish some new Ostrowski type inequalities for functions whose first derivatives in absolute value are logarithmically preinvex. 


\section{Preliminaries}

In this section, we recall some concepts of convexity that are well known in the literature. Throughout this section, $I$ is an interval of $\mathbb{R}$.

Definition 3 (see [18]). A positive function $f: I \rightarrow \mathbb{R}$ is said to be logarithmically convex, if, for all $x, y \in I$ and all $t \in$ $[0,1]$, we have

$$
f(t x+(1-t) y) \leq[f(x)]^{t}[f(y)]^{(1-t)} .
$$

Definition 4 (see [17]). A set $K$ is said to be invex at $x$ with respect to $\eta$, if, for all $x, y \in K$ and $t \in[0,1]$, we have

$$
x+\operatorname{t\eta }(y, x) \in K
$$

$K$ is said to be an invex set with respect to $\eta$ if $K$ is invex at each $x \in K$.

$$
\begin{aligned}
\left|f(x)-\frac{1}{\eta(b, a)} \int_{a}^{a+\eta(b, a)} f(u) d u\right| \leq & \frac{\eta(b, a)\left|f^{\prime}(a)\right|}{2} \\
& \cdot \begin{cases}\left(\left(\frac{x-a}{\eta(b, a)}\right)^{2}+\left(1-\frac{x-a}{\eta(b, a)}\right)^{2}\right) & \text { if } A=1, \\
2\left[\left(2 \frac{x-a}{\eta(b, a)}-1\right) \frac{A^{(x-a) / \eta(b, a)}}{\ln A}+\frac{1-2 A^{(x-a) / \eta(b, a)}+A}{\ln ^{2} A}\right] \quad \text { if } A \neq 1,\end{cases}
\end{aligned}
$$

If $A=1$, then (9) gives

$$
\begin{array}{ccc}
\text { Since } \mid & f^{\prime} \mid \text { is a logarithmically preinvex function, we deduce } & \left|f(x)-\frac{1}{\eta(b, a)} \int_{a}^{a+\eta(b, a)} f(u) d u\right| \\
\mid \begin{aligned}
\left|f(x)-\frac{1}{\eta(b, a)} \int_{a}^{a+\eta(b, a)} f(u) d u\right| \leq \eta(b, a) & \leq \frac{\eta(b, a)\left|f^{\prime}(a)\right|}{2} \\
\cdot\left(\int_{0}^{(x-a) / \eta(b, a)} t\left|f^{\prime}(a)\right|^{(1-t)}\left|f^{\prime}(b)\right|^{t} d t\right. & \cdot\left(\left(\frac{x-a}{\eta(b, a)}\right)^{2}+\left(1-\frac{x-a}{\eta(b, a)}\right)^{2}\right) .
\end{aligned}
\end{array}
$$$$
\begin{aligned}
& \left.+\int_{(x-a) / \eta(b, a)}^{1}(1-t)\left|f^{\prime}(a)\right|^{(1-t)}\left|f^{\prime}(b)\right|^{t} d t\right) \\
& =\eta(b, a)\left|f^{\prime}(a)\right|\left(\int_{0}^{(x-a) / \eta(b, a)} t A^{t} d t\right. \\
& \left.+\int_{(x-a) / \eta(b, a)}^{1}(1-t) A^{t} d t\right) .
\end{aligned}
$$

Theorem 7. Let $K \subseteq[0, \infty)$ be an invex subset with respect to a differentiable function such that $f^{\prime} \in L([a, a+\eta(b, a)])$ and $f^{\prime}(a) \neq 0$. If $\left|f^{\prime}\right|$ is logarithmically preinvex function, then the following inequality holds:

Lemma 6 (see [19]). For $\alpha>0, k>0$, and $z>0$, we have

where $(\alpha)_{i}=\prod_{j=0}^{i-1}(\alpha+j)$.

for all $x \in[a, a+\eta(b, a)]$, where $A=\left|f^{\prime}(b)\right| /\left|f^{\prime}(a)\right|$.

Proof. From Lemma 2 and properties of modulus, we have

$$
\begin{aligned}
& \left|f(x)-\frac{1}{\eta(b, a)} \int_{a}^{a+\eta(b, a)} f(u) d u\right| \leq \eta(b, a) \\
& \quad \cdot\left(\int_{0}^{(x-a) / \eta(b, a)} t\left|f^{\prime}(a+t \eta(b, a))\right| d t\right. \\
& \left.\quad+\int_{(x-a) / \eta(b, a)}^{1}(1-t)\left|f^{\prime}(a+t \eta(b, a))\right| d t\right) .
\end{aligned}
$$


In the case where $A \neq 1$, (9) gives

$$
\begin{gathered}
\left|f(x)-\frac{1}{\eta(b, a)} \int_{a}^{a+\eta(b, a)} f(u) d u\right| \leq \eta(b, a)\left|f^{\prime}(a)\right| \\
\cdot\left(\left(2 \frac{x-a}{\eta(b, a)}-1\right) \frac{A^{(x-a) / \eta(b, a)}}{\ln A}\right. \\
\left.+\frac{1-2 A^{(x-a) / \eta(b, a)}+A}{(\ln A)^{2}}\right),
\end{gathered}
$$

where we use the fact that

$$
\begin{aligned}
& \int_{0}^{(x-a) / \eta(b, a)} t A^{t} \\
& \quad=\frac{x-a}{\eta(b, a)} \frac{A^{(x-a) / \eta(b, a)}}{\ln A}-\frac{A^{(x-a) / \eta(b, a)}-1}{(\ln A)^{2}},
\end{aligned}
$$

$$
\begin{gathered}
\int_{(x-a) / \eta(b, a)}^{1}(1-t) A^{t} d t \\
=-\left(1-\frac{x-a}{\eta(b, a)}\right) \frac{A^{(x-a) / \eta(b, a)}}{\ln A} \\
+\frac{A-A^{(x-a) / \eta(b, a)}}{(\ln A)^{2}} .
\end{gathered}
$$

The desired result follows from (10) and (11).

Corollary 8. In Theorem 7 , if we choose $x=(2 a+\eta(b, a)) / 2$, we obtain the following midpoint inequality:

$$
\begin{array}{r}
\left|f\left(\frac{2 a+\eta(b, a)}{2}\right)-\frac{1}{\eta(b, a)} \int_{a}^{a+\eta(b, a)} f(u) d u\right| \\
\quad \leq \begin{cases}\frac{\eta(b, a)\left|f^{\prime}(a)\right|}{4} & \text { if } A=1, \\
\eta(b, a)\left|f^{\prime}(a)\right|\left[\frac{\sqrt{A}-1}{\ln A}\right]^{2} & \text { if } A \neq 1 .\end{cases}
\end{array}
$$

Corollary 9. Let $f:[a, b] \rightarrow(0, \infty)$ be a differentiable function such that $f^{\prime} \in L([a, b])$ and $f^{\prime}(a) \neq 0$. If $\left|f^{\prime}\right|$ is a logarithmically convex function, then the following inequality holds:

$$
\left|f(x)-\frac{1}{b-a} \int_{a}^{b} f(u) d u\right| \leq \frac{(b-a)\left|f^{\prime}(a)\right|}{2} \begin{cases}\left(\left(\frac{x-a}{b-a}\right)^{2}+\left(\frac{b-x}{b-a}\right)^{2}\right) & \text { if } A=1 \\ 2\left[\left(2 \frac{x-a}{b-a}-1\right) \frac{A^{(x-a) /(b-a)}}{\ln A}+\frac{1-2 A^{(x-a) /(b-a)}+A}{\ln ^{2} A}\right] & \text { if } A \neq 1,\end{cases}
$$

for all $x \in[a, b]$, where $A=\left|f^{\prime}(b)\right| /\left|f^{\prime}(a)\right|$.

Example 10. In Theorem 7 , if we choose $\eta(b, a)=\sqrt{a b}$, the geometric mean, we obtain the following inequality:

$$
\left|f(x)-\frac{1}{\eta(b, a)} \int_{a}^{a+\sqrt{a b}} f(u) d u\right| \leq \frac{\left|f^{\prime}(a)\right| \sqrt{a b}}{2} \begin{cases}\left(\left(\frac{x-a}{\sqrt{a b}}\right)^{2}+\left(1-\frac{x-a}{\sqrt{a b}}\right)^{2}\right) & \text { if } A=1, \\ 2\left[\left(2 \frac{x-a}{\sqrt{a b}}-1\right) \frac{A^{(x-a) / \sqrt{a b}}}{\ln A}+\frac{1-2 A^{(x-a) / \sqrt{a b}}+A}{\ln ^{2} A}\right] & \text { if } A \neq 1 .\end{cases}
$$

Theorem 11. Let $K \subseteq[0, \infty)$ be an invex subset with respect to $\eta: K \times K \rightarrow \mathbb{R}$ and $a, b \in K^{\circ}\left(K^{\circ}\right.$ interior of $\left.K\right)$ with $\eta(b, a)>0$ and $[a, a+\eta(b, a)] \subset K$. Let $f:[a, a+\eta(b, a)] \rightarrow(0, \infty)$ be a differentiable function such that $f^{\prime} \in L([a, a+\eta(b, a)])$ and $f^{\prime}(a) \neq 0$; let $q>1$ with $1 / p+1 / q=1$. If $\left|f^{\prime}\right|^{q}$ is a logarithmically preinvex function, then the following inequality holds: 


$$
\begin{aligned}
\mid f(x) & -\frac{1}{\eta(b, a)} \int_{a}^{a+\eta(b, a)} f(u) d u \mid \\
\leq & \frac{\eta(b, a)\left|f^{\prime}(a)\right|}{(p+1)^{1 / p}} \\
& \cdot \begin{cases}\left(\frac{x-a}{\eta(b, a)}\right)^{2}+\left(1-\frac{x-a}{\eta(b, a)}\right)^{2} & \text { if } A=1, \\
\left(\frac{x-a}{\eta(b, a)}\right)^{1+1 / p}\left(\frac{A^{q((x-a) / \eta(b, a))}-1}{q \ln A}\right)^{1 / q}+\left(1-\frac{x-a}{\eta(b, a)}\right)^{1+1 / p}\left(\frac{A^{q}-A^{q((x-a) / \eta(b, a))}}{q \ln A}\right)^{1 / q} & \text { if } A \neq 1,\end{cases}
\end{aligned}
$$

for all $x \in[a, a+\eta(b, a)]$, where $A=\left|f^{\prime}(b)\right| /\left|f^{\prime}(a)\right|$.

Proof. From Lemma 2, properties of modulus, and Hölder's inequality, we have

$$
\begin{aligned}
& \mid f(x)-\frac{1}{\eta(b, a)} \int_{a}^{a+\eta(b, a)} f(u) d u \mid \leq \eta(b, a) \\
& \cdot\left(\int_{0}^{(x-a) / \eta(b, a)} t^{p} d t\right)^{1 / p} \\
& \cdot\left(\int_{0}^{(x-a) / \eta(b, a)}\left|f^{\prime}(a+t \eta(b, a))\right|^{q} d t\right)^{1 / q} \\
&\left.\left.\left.+\left(\int_{(x-a) / \eta(b, a)}^{1}(1-t)^{p} d t\right)^{1 / p}\right)^{1 / p}\left|f^{\prime}(a+t \eta(b, a))\right|^{q} d t\right)^{1 / q}\right) \\
& \cdot\left(\int_{(x-a) / \eta(b, a)}^{1}\right) \\
&\left.\left.\quad=\frac{\eta(b, a)}{(p+1)^{1 / p}\left(\left(\frac{x-a}{\eta(b, a)}\right)^{1+1 / p}\right.}\left|f_{(x-a) / \eta(b, a)}^{1}(a+t \eta(b, a))\right|^{q} d t\right)^{1 / q}\right) . \\
&+\left(\int_{0}^{(x-a) / \eta(b, a)}\left|f^{\prime}(a+t \eta(b, a))\right|^{q} d t\right)^{1 / q} \\
&+\left(1-\frac{x-a}{\eta(b, a)}\right)^{1+1 / p}
\end{aligned}
$$

Since $\left|f^{\prime}\right|^{q}$ is a logarithmically preinvex function, we deduce

$$
\begin{gathered}
\left|f(x)-\frac{1}{\eta(b, a)} \int_{a}^{a+\eta(b, a)} f(u) d u\right| \\
\leq \frac{\eta(b, a)}{(p+1)^{1 / p}}\left(\left(\frac{x-a}{\eta(b, a)}\right)^{1+1 / p}\right.
\end{gathered}
$$

$$
\begin{aligned}
& \cdot\left(\int_{0}^{(x-a) / \eta(b, a)}\left|f^{\prime}(a)\right|^{q(1-t)}\left|f^{\prime}(b)\right|^{q t} d t\right)^{1 / q} \\
& +\left(1-\frac{x-a}{\eta(b, a)}\right)^{1+1 / p} \\
& \left.\cdot\left(\int_{(x-a) / \eta(b, a)}^{1}\left|f^{\prime}(a)\right|^{q(1-t)}\left|f^{\prime}(b)\right|^{q t} d t\right)^{1 / q}\right) \\
& =\frac{\eta(b, a)\left|f^{\prime}(a)\right|}{(p+1)^{1 / p}}\left(\left(\frac{x-a}{\eta(b, a)}\right)^{1+1 / p}\right. \\
& \cdot\left(\int_{0}^{(x-a) / \eta(b, a)} A^{q t} d t\right)^{1 / q}+\left(1-\frac{x-a}{\eta(b, a)}\right)^{1+1 / p} \\
& \left.\cdot\left(\int_{(x-a) / \eta(b, a)}^{1} A^{q t} d t\right)^{1 / q}\right) .
\end{aligned}
$$

If $A=1$, then (18) gives

$$
\begin{aligned}
& \left|f(x)-\frac{1}{\eta(b, a)} \int_{a}^{a+\eta(b, a)} f(u) d u\right| \\
& \leq \frac{\eta(b, a)\left|f^{\prime}(a)\right|}{(p+1)^{1 / p}}\left(\left(\frac{x-a}{\eta(b, a)}\right)^{2}+\left(1-\frac{x-a}{\eta(b, a)}\right)^{2}\right) .
\end{aligned}
$$

In the case where $A \neq 1$, (18) becomes

$$
\begin{aligned}
& \left|f(x)-\frac{1}{\eta(b, a)} \int_{a}^{a+\eta(b, a)} f(u) d u\right| \\
& \quad \leq \frac{\eta(b, a)\left|f^{\prime}(a)\right|}{(p+1)^{1 / p}}\left(\left(\frac{x-a}{\eta(b, a)}\right)^{1+1 / p}\right. \\
& \quad \cdot\left(\frac{A^{q((x-a) / \eta(b, a))}-1}{q \ln A}\right)^{1 / q}+\left(1-\frac{x-a}{\eta(b, a)}\right)^{1+1 / p} \\
& \left.\quad \cdot\left(\frac{A^{q}-A^{q((x-a) / \eta(b, a))}}{q \ln A}\right)^{1 / q}\right),
\end{aligned}
$$


where we use the fact that

$$
\begin{aligned}
& \int_{0}^{(x-a) / \eta(b, a)} A^{q t} d t=\frac{A^{q((x-a) / \eta(b, a))}-1}{q \ln A}, \\
& \int_{(x-a) / \eta(b, a)}^{1} A^{q t} d t=\frac{A^{q}-A^{q((x-a) / \eta(b, a))}}{q \ln A} .
\end{aligned}
$$

From (19) and (20), we get the desired result.

Corollary 12. In Theorem 11, if we choose $x=(2 a+\eta(b, a)) / 2$, we obtain the following midpoint inequality:

$$
\left|f\left(\frac{2 a+\eta(b, a)}{2}\right)-\frac{1}{\eta(b, a)} \int_{a}^{a+\eta(b, a)} f(u) d u\right|
$$

$$
\begin{aligned}
& \left|f(x)-\frac{1}{b-a} \int_{a}^{b} f(u) d u\right| \\
& \quad \leq \frac{(b-a)\left|f^{\prime}(a)\right|}{(p+1)^{1 / p}} \begin{cases}\left(\frac{x-a}{b-a}\right)^{2}+\left(\frac{b-x}{b-a}\right)^{2} & \text { if } A=1, \\
\left(\frac{x-a}{b-a}\right)^{1+1 / p}\left(\frac{A^{q((x-a) /(b-a))}-1}{q \ln A}\right)^{1 / q}+\left(\frac{b-x}{b-a}\right)^{1+1 / p}\left(\frac{A^{q}-A^{q((x-a) /(b-a))}}{q \ln A}\right)^{1 / q} \text { if } A \neq 1,\end{cases}
\end{aligned}
$$

Corollary 13. Let $f:[a, b] \rightarrow(0, \infty)$ be a differentiable function such that $f^{\prime} \in L([a, b])$ and $f^{\prime}(a) \neq 0$; let $q>1$ with $1 / p+1 / q=1$. If $\left|f^{\prime}\right|^{q}$ is a logarithmically convex function, then the following inequality holds:

for all $x \in[a, b]$, where $A=\left|f^{\prime}(b)\right| /\left|f^{\prime}(a)\right|$.

Example 14. In Theorem 11, if we choose $\eta(b, a)=(a+b) / 2$, the arithmetic mean, we obtain the following inequality:

$$
\begin{aligned}
\mid f(x) & -\frac{2}{a+b} \int_{a}^{a+(a+b) / 2} f(u) d u \mid \\
\leq & \frac{(a+b)\left|f^{\prime}(a)\right|}{2(p+1)^{1 / p}} \\
& \cdot\left\{\frac{4}{(a+b)^{2}}\left[(x-a)^{2}+\left(\frac{b+3 a-2 x}{2}\right)^{2}\right]\right. \\
\left(2 \frac{x-a}{a+b}\right)^{1+1 / p}\left(\frac{A^{2 q((x-a) /(a+b))}-1}{q \ln A}\right)^{1 / q}+\left(\frac{b+3 a-2 x}{2}\right)^{1+1 / p}\left(\frac{A^{q}-A^{2 q((x-a) /(a+b))}}{q \ln A}\right)^{1 / q} & \text { if } A=1,
\end{aligned}
$$

Theorem 15. Let $K \subseteq[0, \infty)$ be an invex subset with respect to $\eta: K \times K \rightarrow \mathbb{R}$ and $a, b \in K^{\circ}\left(K^{\circ}\right.$ interior of $\left.K\right)$ with $\eta(b, a)>$ 0 and $[a, a+\eta(b, a)] \subset K$. Let $f:[a, a+\eta(b, a)] \rightarrow(0, \infty)$ be a differentiable function such that $f^{\prime} \in L([a, a+\eta(b, a)])$ and $f^{\prime}(a) \neq 0$; let $q>1$. If $\left|f^{\prime}\right|^{q}$ is a logarithmically preinvex function, then the following inequality holds:

$$
\begin{aligned}
& \left|f(x)-\frac{1}{\eta(b, a)} \int_{a}^{a+\eta(b, a)} f(u) d u\right| \leq \frac{\eta(b, a)}{2^{1-1 / q}}\left|f^{\prime}(a)\right| \\
& \qquad\left\{\begin{array}{l}
\frac{1}{2^{1 / q}}\left(\left(\frac{x-a}{\eta(b, a)}\right)^{2}+\left(1-\frac{x-a}{\eta(b, a)}\right)^{2}\right) \\
\left(\left(\frac{x-a}{\eta(b, a)}\right)^{2-2 / q}\left(\frac{x-a}{\eta(b, a)} \frac{A^{q((x-a) / \eta(b, a))}}{\ln A}+\frac{1-A^{q((x-a) / \eta(b, a))}}{\ln ^{2} A}\right)^{1 / q}+\left(1-\frac{x-a}{\eta(b, a)}\right)^{2-2 / q}\left(\frac{A^{q}-A^{q((x-a) / \eta(b, a))}}{\ln ^{2} A}-\left(1-\frac{x-a}{\eta(b, a)}\right) \frac{A^{q((x-a) / \eta(b, a))}}{\ln A}\right)^{1 / q}\right) \text { if } A \neq 1,
\end{array}\right.
\end{aligned}
$$

for all $x \in[a, a+\eta(b, a)]$, where $A=\left|f^{\prime}(b)\right| /\left|f^{\prime}(a)\right|$. 
Proof. From Lemma 2, properties of modulus, and power mean inequality, we have

$$
\begin{aligned}
\mid f & (x)-\frac{1}{\eta(b, a)} \int_{a}^{a+\eta(b, a)} f(u) d u \mid \leq \eta(b, a) \\
& \cdot\left(\left(\int_{0}^{(x-a) / \eta(b, a)} t d t\right)^{1-1 / q}\right. \\
\cdot & \left(\int_{0}^{(x-a) / \eta(b, a)} t\left|f^{\prime}(a+t \eta(b, a))\right|^{q} d t\right)^{1 / q} \\
+ & \left(\int_{(x-a) / \eta(b, a)}^{1}(1-t) d t\right)^{1-1 / q} \\
\cdot & \left.\left(\int_{(x-a) / \eta(b, a)}^{1}(1-t)\left|f^{\prime}(a+t \eta(b, a))\right|^{q} d t\right)^{1 / q}\right) \\
& =\frac{\eta(b, a)}{2^{1-1 / q}\left(\left(\frac{x-a}{\eta(b, a)}\right)^{2(1-1 / q)}\right.} \\
& \cdot\left(\int_{0}^{(x-a) / \eta(b, a)} t\left|f^{\prime}(a+t \eta(b, a))\right|^{q} d t\right)^{1 / q} \\
+ & \left(1-\frac{x-a}{\eta(b, a)}\right)^{2(1-1 / q)} \\
\cdot & \left.\left(\int_{(x-a) / \eta(b, a)}^{1}(1-t)\left|f^{\prime}(a+t \eta(b, a))\right|^{q} d t\right)^{1 / q}\right) .
\end{aligned}
$$

Since $\left|f^{\prime}\right|^{q}$ is a logarithmically preinvex function, we deduce

$$
\begin{aligned}
& \left|f(x)-\frac{1}{\eta(b, a)} \int_{a}^{a+\eta(b, a)} f(u) d u\right| \leq \frac{\eta(b, a)}{2^{1-1 / q}}\left|f^{\prime}(a)\right| \\
& \quad \cdot\left(\left(\frac{x-a}{\eta(b, a)}\right)^{2-2 / q}\left(\int_{0}^{(x-a) / \eta(b, a)} t A^{q t} d t\right)^{1 / q}\right. \\
& \quad+\left(1-\frac{x-a}{\eta(b, a)}\right)^{2-2 / q} \\
& \left.\quad \cdot\left(\int_{(x-a) / \eta(b, a)}^{1}(1-t) A^{q t} d t\right)^{1 / q}\right) .
\end{aligned}
$$

$$
\begin{aligned}
& \left|f\left(\frac{2 a+\eta(b, a)}{2}\right)-\frac{1}{\eta(b, a)} \int_{a}^{a+\eta(b, a)} f(u) d u\right| \\
& \quad \leq \frac{\eta(b, a)}{4}\left|f^{\prime}(a)\right|\left\{\begin{array}{l}
1 \\
\frac{1}{2^{1-3 / q}}\left(\left(\frac{1-A^{q / 2}}{\ln ^{2} A}+\frac{A^{q / 2}}{2 \ln A}\right)^{1 / q}+A^{1 / 2}\left(\frac{A^{q / 2}-1}{\ln ^{2} A}-\frac{1}{2 \ln A}\right)^{1 / q}\right) \text { if } A \neq 1 .
\end{array}\right.
\end{aligned}
$$

For $A \neq 1$, (27) gives

$$
\begin{aligned}
& \left|f(x)-\frac{1}{\eta(b, a)} \int_{a}^{a+\eta(b, a)} f(u) d u\right| \leq \frac{\eta(b, a)}{2^{1-1 / q}}\left|f^{\prime}(a)\right| \\
& \cdot\left(( \frac { x - a } { \eta ( b , a ) } ) ^ { 2 - 2 / q } \left(\frac{x-a}{\eta(b, a)} \frac{A^{q((x-a) / \eta(b, a))}}{\ln A}\right.\right. \\
& \left.+\frac{1-A^{q((x-a) / \eta(b, a))}}{\ln ^{2} A}\right)^{1 / q}+\left(1-\frac{x-a}{\eta(b, a)}\right)^{2-2 / q} \\
& \left.\cdot\left(\frac{A^{q}-A^{q((x-a) / \eta(b, a))}}{\ln ^{2} A}\right)^{1 / q}\right) \\
& -\left(1-\frac{x-a}{\eta(b, a)}\right) \frac{A^{q((x-a) / \eta(b, a))}}{\ln A}
\end{aligned}
$$

where we use the fact that

$$
\begin{aligned}
& \int_{0}^{(x-a) / \eta(b, a)} t A^{q t} d t \\
& =\frac{x-a}{\eta(b, a)} \frac{A^{q(x-a) / \eta(b, a)}}{\ln A}+\frac{1-A^{q(x-a) / \eta(b, a)}}{\ln ^{2} A}, \\
& \int_{(x-a) / \eta(b, a)}^{1}(1-t) A^{q t} d t \\
& =\frac{A^{q}-A^{q((x-a) / \eta(b, a))}}{\ln ^{2} A} \\
& -\left(1-\frac{x-a}{\eta(b, a)}\right) \frac{A^{q((x-a) / \eta(b, a))}}{\ln A} .
\end{aligned}
$$

From (28) and (29), we obtain the desired result.

Corollary 16. In Theorem 15 , if we choose $x=(2 a+\eta(b, a)) / 2$, we obtain the following midpoint inequality: 
Corollary 17. Let $f:[a, a+\eta(b, a)] \rightarrow(0, \infty)$ be $a$ differentiable function such that $f^{\prime} \in L([a, a+\eta(b, a)])$ and $f^{\prime}(a) \neq 0$; let $q>1$. If $\left|f^{\prime}\right|^{q}$ is a logarithmically convex function, then the following inequality holds:

$$
\begin{aligned}
& \left|f(x)-\frac{1}{b-a} \int_{a}^{b} f(u) d u\right| \leq \frac{(b-a)}{2^{1-1 / q}}\left|f^{\prime}(a)\right| \\
& \qquad\left\{\begin{array}{l}
\frac{1}{2^{1 / q}}\left(\left(\frac{x-a}{b-a}\right)^{2}+\left(\frac{b-x}{b-a}\right)^{2}\right) \\
\left(\left(\frac{x-a}{b-a}\right)^{2-2 / q}\left(\frac{x-a}{b-a} \frac{A^{q((x-a) /(b-a))}}{\ln A}+\frac{1-A^{q((x-a) /(b-a))}}{\ln ^{2} A}\right)^{1 / q}+\left(\frac{b-x}{b-a}\right)^{2-2 / q}\left(\frac{A^{q}-A^{q((x-a) /(b-a))}}{\ln ^{2} A}-\left(\frac{b-x}{b-a}\right) \frac{A^{q((x-a) /(b-a))}}{\ln A}\right)^{1 / q}\right) \text { if } A \neq 1,
\end{array}\right.
\end{aligned}
$$

for all $x \in[a, b]$, where $A=\left|f^{\prime}(b)\right| /\left|f^{\prime}(a)\right|$.

Example 18. In Theorem 15 , if we choose $\eta(b, a)=(b-$ $a) /(\ln b-\ln a)$ with $a \neq b$, the logarithmic mean, we obtain the following inequality:

$$
\begin{aligned}
\mid f(x) & -\frac{\ln b-\ln a}{b-a} \int_{a}^{a+(b-a) /(\ln b-\ln a)} f(u) d u \mid \\
\leq & \frac{b-a}{2^{1-1 / q}(\ln b-\ln a)}\left|f^{\prime}(a)\right| \\
& \cdot \begin{cases}\frac{1}{2^{1 / q}}\left(\theta^{2}+(1-\theta)^{2}\right) & \text { if } A=1, \\
\left(\theta^{2-2 / q}\left(\theta \frac{A^{q \theta}}{\ln A}+\frac{1-A^{q \theta}}{\ln ^{2} A}\right)^{1 / q}+(1-\theta)^{2-2 / q}\left(\frac{A^{q}-A^{q \theta}}{\ln ^{2} A}-(1-\theta) \frac{A^{q \theta}}{\ln A}\right)^{1 / q}\right) & \text { if } A \neq 1,\end{cases}
\end{aligned}
$$

where $\theta=(x-a)(\ln b-\ln a) /(b-a)$.

Theorem 19. Suppose that all the assumptions of Theorem 15 are satisfied, then the following inequality holds:

$$
\begin{aligned}
& \left|f(x)-\frac{1}{\eta(b, a)} \int_{a}^{a+\eta(b, a)} f(u) d u\right| \leq \eta(b, a)\left|f^{\prime}(a)\right| \\
& .\left\{\begin{array}{l}
\frac{1}{(q+1)^{1 / q}}\left(\left(\frac{x-a}{\eta(b, a)}\right)^{2}+\left(1-\frac{x-a}{\eta(b, a)}\right)^{2}\right) \\
\left.A^{(x-a) / \eta(b, a)}\left(\left(1-\frac{x-a}{\eta(b, a)}\right) \frac{x-a}{\eta(b, a)}\left(\sum_{i=1}^{\infty} \frac{\left(\ln A^{q(1-(x-a) / \eta(b, a))}\right)^{i-1}}{(\alpha)_{i}}\right)^{1 / q}+\left(\frac{x-a}{\eta(b, a)}\right)^{2}\left(\sum_{i=1}^{\infty} \frac{\left(-\ln A^{q((x-a) / \eta(b, a))}\right)^{i-1}}{(\alpha)_{i}}\right) \text { if }\right)^{1 / q}\right) \text { if } A \neq 1,
\end{array}\right.
\end{aligned}
$$

for all $x \in[a, a+\eta(b, a)]$, where $A=\left|f^{\prime}(b)\right| /\left|f^{\prime}(a)\right|$ and $(q+$ $1)_{i}=\prod_{j=0}^{i-1}(q+1+j)$.

Proof. From Lemma 2, properties of modulus, and power mean inequality, we have

$$
\begin{gathered}
\left|f(x)-\frac{1}{\eta(b, a)} \int_{a}^{a+\eta(b, a)} f(u) d u\right| \leq \eta(b, a) \\
\cdot\left(\left(\int_{0}^{(x-a) / \eta(b, a)} d t\right)^{1-1 / q}\right.
\end{gathered}
$$

$$
\begin{aligned}
& \cdot\left(\int_{0}^{(x-a) / \eta(b, a)} t^{q}\left|f^{\prime}(a+t \eta(b, a))\right|^{q} d t\right)^{1 / q} \\
& +\left(\int_{(x-a) / \eta(b, a)}^{1} d t\right)^{1-1 / q}\left(\int_{(x-a) / \eta(b, a)}^{1}(1-t)^{q}\right.
\end{aligned}
$$$$
\left.\left.\cdot\left|f^{\prime}(a+t \eta(b, a))\right|^{q} d t\right)^{1 / q}\right)=\eta(b, a)
$$$$
\cdot\left(\left(\frac{x-a}{\eta(b, a)}\right)^{1-1 / q}\right.
$$ 


$$
\begin{aligned}
& \cdot\left(\int_{0}^{(x-a) / \eta(b, a)} t^{q}\left|f^{\prime}(a+t \eta(b, a))\right|^{q} d t\right)^{1 / q}+(1 \\
& \left.-\frac{x-a}{\eta(b, a)}\right)^{1-1 / q}\left(\int_{(x-a) / \eta(b, a)}^{1}(1-t)^{q}\right. \\
& \left.\left.\cdot\left|f^{\prime}(a+t \eta(b, a))\right|^{q} d t\right)^{1 / q}\right) .
\end{aligned}
$$

Since $\left|f^{\prime}\right|^{q}$ is a logarithmically preinvex function, we deduce

$$
\begin{aligned}
& \left|f(x)-\frac{1}{\eta(b, a)} \int_{a}^{a+\eta(b, a)} f(u) d u\right| \leq \eta(b, a)\left|f^{\prime}(a)\right| \\
& \quad \cdot\left(\left(\frac{x-a}{\eta(b, a)}\right)^{1-1 / q}\left(\int_{0}^{(x-a) / \eta(b, a)} t^{q} A^{q t}\right)^{1 / q}\right. \\
& \quad+\left(1-\frac{x-a}{\eta(b, a)}\right)^{1-1 / q} \\
& \left.\quad \cdot\left(\int_{(x-a) / \eta(b, a)}^{1}(1-t)^{q} A^{q t} d t\right)^{1 / q}\right) .
\end{aligned}
$$

If $A=1,(36)$ gives

$$
\begin{aligned}
\left|f(x)-\frac{1}{\eta(b, a)} \int_{a}^{a+\eta(b, a)} f(u) d u\right| \\
\leq \frac{\eta(b, a)\left|f^{\prime}(a)\right|}{(q+1)^{1 / q}} \\
\\
\quad \cdot\left(\left(\frac{x-a}{\eta(b, a)}\right)^{2}+\left(1-\frac{x-a}{\eta(b, a)}\right)^{2}\right) .
\end{aligned}
$$

In the case where $A \neq 1$, we can restate (36) as

$$
\begin{gathered}
\left|f(x)-\frac{1}{\eta(b, a)} \int_{a}^{a+\eta(b, a)} f(u) d u\right| \leq \eta(b, a)\left|f^{\prime}(a)\right| \\
\cdot\left(\left(\frac{x-a}{\eta(b, a)}\right)^{2}\left(\int_{0}^{1} t^{q}\left(A^{q((x-a) / \eta(b, a))}\right)^{t} d t\right)^{1 / q}\right.
\end{gathered}
$$

$$
\begin{aligned}
& +\left(1-\frac{x-a}{\eta(b, a)}\right) \frac{x-a}{\eta(b, a)} A^{(x-a) / \eta(b, a)} \\
& \left.\cdot\left(\int_{0}^{1}(t-1)^{q}\left(A^{q(1-(x-a) / \eta(b, a))}\right)^{t} d t\right)^{1 / q}\right) .
\end{aligned}
$$

Applying Lemma 6 with $z=1$, we get

$$
\begin{aligned}
& \int_{0}^{1} t^{q}\left(A^{q((x-a) / \eta(b, a))}\right)^{t} d t \\
& \quad=A^{q((x-a) / \eta(b, a))} \sum_{i=1}^{\infty} \frac{\left(-\ln A^{q((x-a) / \eta(b, a))}\right)^{i-1}}{(\alpha)_{i}} \\
& \int_{0}^{1}(t-1)^{q}\left(A^{q(1-(x-a) / \eta(b, a))}\right)^{t} d t \\
& =\sum_{i=1}^{\infty} \frac{\left(\ln A^{q(1-(x-a) / \eta(b, a))}\right)^{i-1}}{(\alpha)_{i}} .
\end{aligned}
$$

Substituting (39) into (38), we obtain

$$
\begin{aligned}
\mid f & (x)-\frac{1}{\eta(b, a)} \int_{a}^{a+\eta(b, a)} f(u) d u|\leq \eta(b, a)| f^{\prime}(a) \mid \\
& \cdot\left(\left(\frac{x-a}{\eta(b, a)}\right)^{2}\right. \\
& \cdot A^{(x-a) / \eta(b, a)}\left(\sum_{i=1}^{\infty} \frac{\left(-\ln A^{q((x-a) / \eta(b, a))}\right)^{i-1}}{(\alpha)_{i}}\right)^{1 / q} \\
& +\left(1-\frac{x-a}{\eta(b, a)}\right) \frac{x-a}{\eta(b, a)} A^{(x-a) / \eta(b, a)} \\
& \left.\cdot\left(\sum_{i=1}^{\infty} \frac{\left(\ln A^{q(1-(x-a) / \eta(b, a))}\right)^{i-1}}{(\alpha)_{i}}\right)^{1 / q}\right) .
\end{aligned}
$$

The desired result follows from (37) and (40).

Corollary 20. In Theorem 19, if we choose $x=(2 a+\eta(b, a)) / 2$, we obtain the following midpoint inequality: 


$$
\begin{aligned}
\mid f\left(\frac{2 a+\eta(b, a)}{2}\right)- & \frac{1}{\eta(b, a)} \int_{a}^{a+\eta(b, a)} f(u) d u \mid \\
& \leq \frac{\eta(b, a)\left|f^{\prime}(a)\right|}{2} \begin{cases}\frac{1}{(q+1)^{1 / q}} & \text { if } A=1, \\
\frac{\sqrt{A}}{2}\left(\left(\sum_{i=1}^{\infty} \frac{\left(\ln A^{q / 2}\right)^{i-1}}{(\alpha)_{i}}\right)^{1 / q}+\left(\sum_{i=1}^{\infty} \frac{\left(-\ln A^{q / 2}\right)^{i-1}}{(\alpha)_{i}}\right)^{1 / q}\right) & \text { if } A \neq 1 .\end{cases}
\end{aligned}
$$

Corollary 21. In Theorem 19, if we choose $\eta(b, a)=b-a$, we obtain the following inequality:

$$
\begin{aligned}
& \left|f(x)-\frac{1}{b-a} \int_{a}^{b} f(u) d u\right| \leq(b-a)\left|f^{\prime}(a)\right| \\
& \begin{cases}\frac{1}{(q+1)^{1 / q}}\left(\left(\frac{x-a}{b-a}\right)^{2}+\left(\frac{b-x}{b-a}\right)^{2}\right) & \text { if } A=1, \\
A^{(x-a) /(b-a)}\left(\frac{(b-x)(x-a)}{(b-a)^{2}}\left(\sum_{i=1}^{\infty} \frac{\left(\ln A^{q((b-x) /(b-a))}\right)^{i-1}}{(\alpha)_{i}}\right)^{1 / q}+\left(\frac{x-a}{b-a}\right)^{2}\left(\sum_{i=1}^{\infty} \frac{\left(-\ln A^{q((x-a) /(b-a))}\right)^{i-1}}{(\alpha)_{i}}\right)\right. & \text { if } A \neq 1 .\end{cases}
\end{aligned}
$$

Remark 22. In all the above theorems, inequalities for nonconvex functions could be drawn by just replacing $\eta(b, a)$ by other means than those in the previously mentioned examples.

\section{Competing Interests}

The author declares that they have no competing interests.

\section{References}

[1] D. S. Mitrinović, J. E. Pečarić, and A. M. Fink, Classical and New Inequalities in Analysis, vol. 61 of Mathematics and Its Applications (East European Series), Kluwer Academic Publishers Group, Dordrecht, The Netherlands, 1993.

[2] M. W. Alomari, M. E. Özdemir, and H. Kavurmac, "On companion of Ostrowski inequality for mappings whose first derivatives absolute value are convex with applications," Miskolc Mathematical Notes, vol. 13, no. 2, pp. 233-248, 2012.

[3] N. S. Barnett, P. Cerone, S. S. Dragomir, M. R. Pinheiro, and A. Sofo, "Ostrowski type inequalities for functions whose modulus of derivatives are convex and applications," RGMIA Research Report Collection, vol. 5, no. 2, article 1, 2002.

[4] P. Cerone and S. S. Dragomir, "Ostrowski type inequalities for functions whose derivatives satisfy certain convexity assumptions," Demonstratio Mathematica, vol. 37, no. 2, pp. 299-308, 2004.

[5] I. Işcan, "Ostrowski type inequalities for functions whose derivatives are preinvex," Bulletin of the Iranian Mathematical Society, vol. 40, no. 2, pp. 373-386, 2014.
[6] M. E. Kiris and M. Z. Sarikaya, "On Ostrowski type inequalities and Čebyšev type inequalities with applications," Filomat, vol. 29, no. 8, pp. 1695-1713, 2015.

[7] M. A. Noor, K. I. Noor, and M. U. Awan, "Some quantum integral inequalities via preinvex functions," Applied Mathematics and Computation, vol. 269, pp. 242-251, 2015.

[8] M. A. Noor, K. I. Noor, and M. U. Awan, "Fractional Ostrowski inequalities for $(s, m)$-Godunova-Levin functions," Facta Universitatis, Series: Mathematics and Informatics, vol. 30, no. 4, pp. 489-499, 2015.

[9] E. Set, M. E. Özdemir, M. Z. Sarikaya, and M. Z. Sarıkaya, "New inequalities of Ostrowski's type for s-convex functions in the second sense with applications," Facta Universitatis, Series: Mathematics and Informatics, vol. 27, no. 1, pp. 67-82, 2012.

[10] E. Set, "New inequalities of Ostrowski type for mappings whose derivatives are s-convex in the second sense via fractional integrals," Computers \& Mathematics with Applications, vol. 63, no. 7, pp. 1147-1154, 2012.

[11] M. A. Hanson, "On sufficiency of the Kuhn-Tucker conditions," Journal of Mathematical Analysis and Applications, vol. 80, no. 2, pp. 545-550, 1981.

[12] A. Ben-Israel and B. Mond, "What is invexity?" The Journal of the Australian Mathematical Societ-Series B: Applied Mathematics, vol. 28, no. 1, pp. 1-9, 1986.

[13] R. Pini, "Invexity and generalized convexity", Optimization, vol. 22, no. 4, pp. 513-525, 1991.

[14] M. A. Noor, "Variational-like inequalities," Optimization, vol. 30, no. 4, pp. 323-330, 1994.

[15] M. A. Noor, "Invex equilibrium problems," Journal of Mathematical Analysis and Applications, vol. 302, no. 2, pp. 463-475, 2005. 
[16] X. M. Yang and D. Li, "On properties of preinvex functions," Journal of Mathematical Analysis and Applications, vol. 256, no. 1, pp. 229-241, 2001.

[17] T. Weir and B. Mond, "Pre-invex functions in multiple objective optimization," Journal of Mathematical Analysis and Applications, vol. 136, no. 1, pp. 29-38, 1988.

[18] J. E. Pečarić, F. Proschan, and Y. L. Tong, Convex Functions, Partial Orderings, and Statistical Applications, vol. 187 of Mathematics in Science and Engineering, Academic Press, Boston, Mass, USA, 1992.

[19] J. Wang, J. Deng, and M. Fečkan, "Hermite-Hadamard-type inequalities for $r$-convex functions based on the use of Riemann-Liouville fractional integrals," Ukrainian Mathematical Journal, vol. 65, no. 2, pp. 193-211, 2013. 


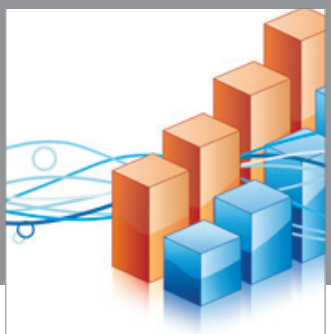

Advances in

Operations Research

vatem alat4

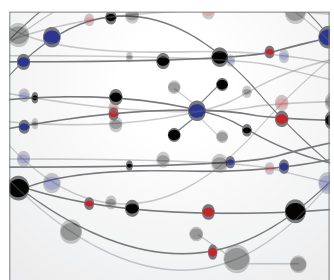

\section{The Scientific} World Journal
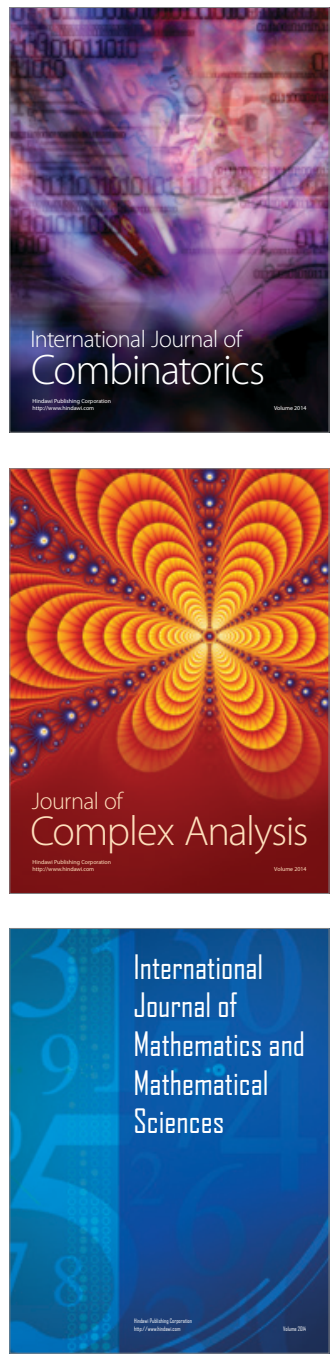
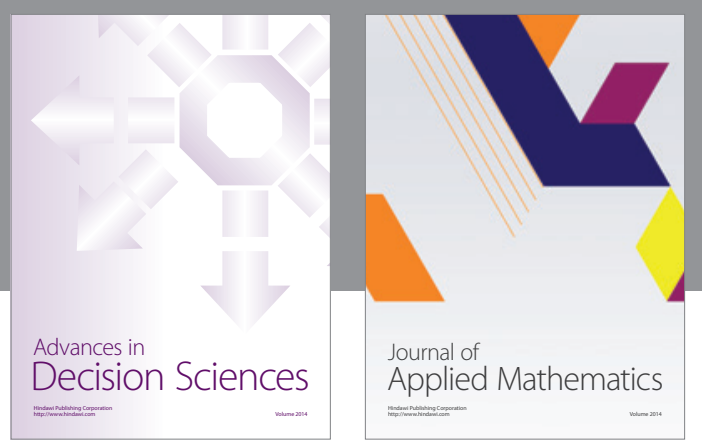

Algebra

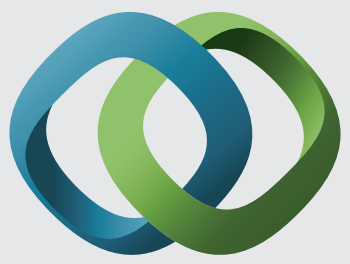

\section{Hindawi}

Submit your manuscripts at

http://www.hindawi.com
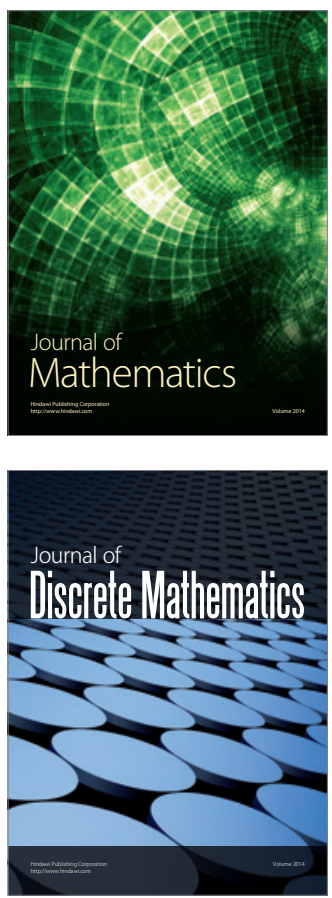

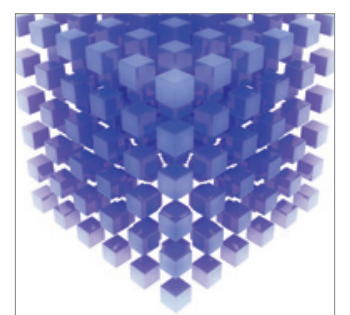

Mathematical Problems in Engineering
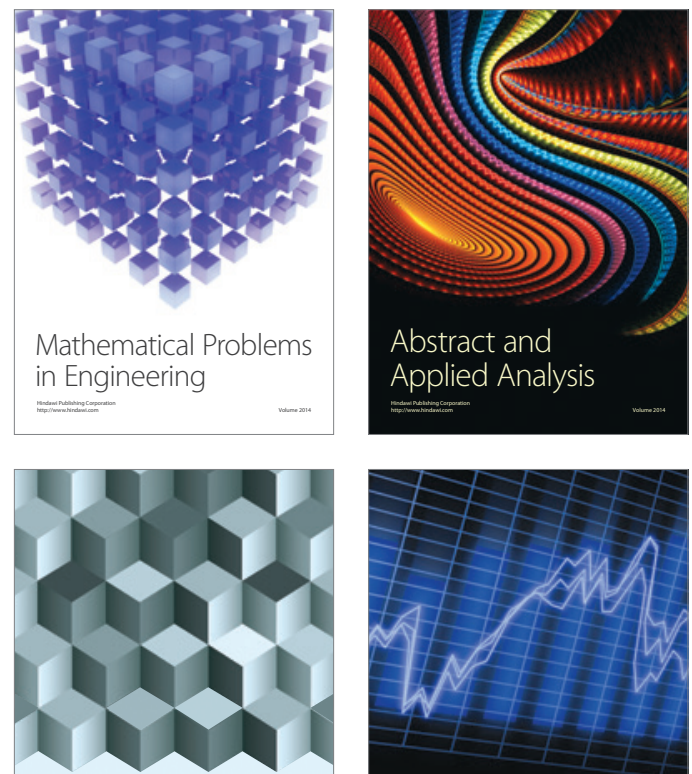

Journal of

Function Spaces

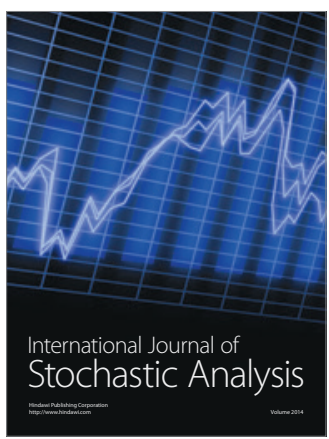

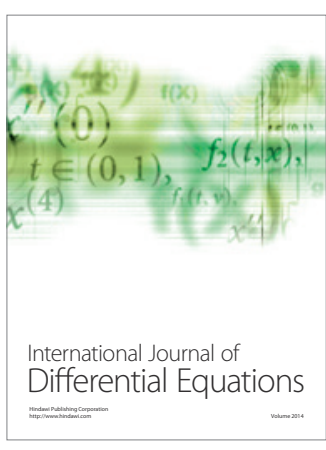
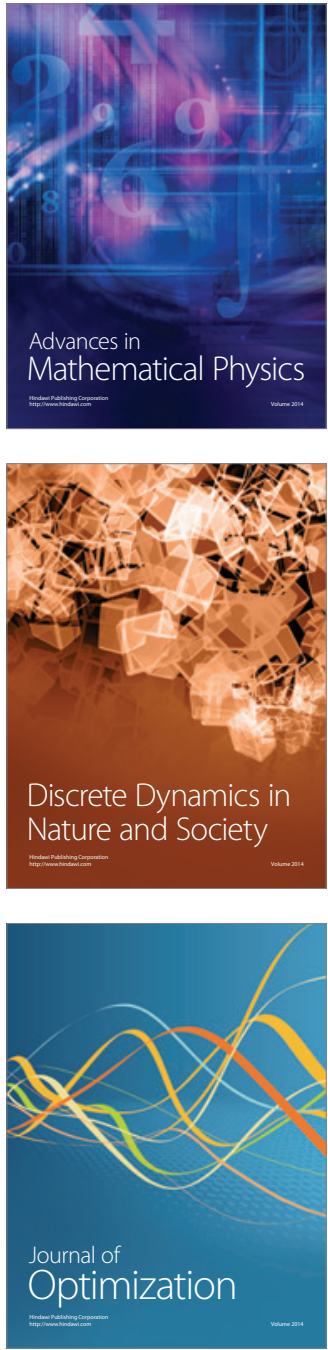